\title{
Intra-tumoural lipid composition and lymphovascular invasion in breast cancer via non-invasive magnetic resonance spectroscopy
}

\author{
Sai Man Cheung ${ }^{1}$ (D) Ehab Husain ${ }^{2} \cdot$ Vasiliki Mallikourti $^{1} \cdot$ Yazan Masannat $^{3} \cdot$ Steven Heys $^{3} \cdot$ Jiabao He $^{1}$
}

Received: 6 May 2020 / Revised: 7 October 2020 / Accepted: 11 November 2020 / Published online: 3 December 2020

(C) The Author(s) 2020

\begin{abstract}
Objectives Despite improved survival due to new treatments, the 10-year survival rate in patients with breast cancer is approximately 75\%. Lymphovascular invasion (LVI), a prognostic marker independent from histological grade and stage, can only be fully determined at final histological examination. Lipid composition is deregulated in tumour via de novo lipogenesis, with alteration in lipogenic genes in LVI. We hypothesise alteration in lipid composition derived from novel non-invasive spectroscopy method is associated with LVI positivity.

Methods Thirty female patients (age 39-78) with invasive ductal carcinoma were enrolled, with 13 LVI negative and 17 LVI positive. Saturated, monounsaturated, polyunsaturated fatty acids and triglycerides (SFA, MUFA, PUFA and TRG) were quantified from ex vivo breast tumours freshly excised from patients on a $3 \mathrm{~T}$ clinical MRI scanner, and proliferative activity marker Ki-67 and serotonin derived histologically.

Results There were significantly lower MUFA $(p=0.0189)$ in LVI positive (median: 0.37 , interquartile range (IQR): $0.25-0.64)$ than negative $(0.63,0.49-0.96)$. There were significantly lower TRG $(p=0.0226)$ in LVI positive $(1.32,0.95-2.43)$ than negative $(2.5$, $1.92-4.15)$. There was no significant difference in SFA $(p=0.6009)$ or PUFA $(p=0.1641)$. There was no significant correlation between lipid composition against Ki-67 or serotonin, apart from a borderline negative correlation between PUFA and serotonin $(r=-$ $0.3616, p=0.0496$ ).

Conclusion Lipid composition might provide a biomarker to study lymphovascular invasion in breast cancer.

Key Points

- Monounsaturated fatty acids in lymphovascular invasion (LVI) positive invasive breast carcinoma were significantly lower than that in LVI negative.

- Triglycerides in LVI positive invasive breast carcinoma were significantly lower than that in LVI negative.

- Lipid composition from MR spectroscopy reflects the rate of de novo lipogenesis and provides a potential biomarker independent from histological grade and stage.
\end{abstract}

Keywords Monounsaturated fatty acids (MUFA) $\cdot$ Triglycerides $\cdot$ Saturated fatty acids (SFA) $\cdot$ Serotonin $\cdot$ Magnetic resonance spectroscopy (MRS)

Abbreviations

COSY Correlation spectroscopy

DQF Double quantum filtering

Sai Man Cheung

g.cheung@abdn.ac.uk

1 Institute of Medical Sciences, School of Medicine, University of Aberdeen, Foresterhill, Aberdeen AB25 2ZD, UK

2 Pathology Department, Aberdeen Royal Infirmary, Aberdeen, UK

3 Breast Unit, Aberdeen Royal Infirmary, Aberdeen, UK
IQR Interquartile range

LVI Lymphovascular invasion

MRS Magnetic resonance spectroscopy

MUFA Monounsaturated fatty acids

NPI Nottingham prognostic index

ppm Parts per million

PRESS Point resolved spectroscopy

PUFA Polyunsaturated fatty acids

SFA Saturated fatty acids

TE Time of echo

TR Time of repetition

TRG Triglycerides 


\section{Introduction}

The 10-year survival rate in patients with breast cancer is approximately $75 \%$ [1], despite the improved survival [1] as a result of early detection, screening and new treatments [2]. Lymphovascular invasion (LVI) is an important histopathological feature of prognostic value independent from histological grade [3] that correlates with proliferative activity marker Ki-67 [4] and nodal status [5]. However, the full assessment of LVI is achieved at the final histology [6] due to partial sampling error in preoperative biopsy [7]. Other factors including tissue shrinkage (although immunohistochemistry might be used to highlight endothelial cells) [8] and mechanical forceinduced cell displacement [9] can affect the assessment, especially after tumour dissipation induced by neoadjuvant chemotherapy can make histological assessment of LVI difficult. LVI is associated with elevated risk of lymph node metastasis [10] through tumour-associated lymphangiogenesis [11], and the expression alterations of genes in the incorporation of saturated- and monounsaturated fatty acids (SFA, MUFA) in phospholipid metabolism [12]. Pro-inflammatory polyunsaturated fatty acid (PUFA)-derived eicosanoids recruit macrophages to trigger immune response leading to elevated serotonin (5-HT) [13] that is associated with worse 10-year survival [14]. Hence, non-invasive quantification of lipid composition as a marker of LVI has a potential role in personalised care, especially in the context of neoadjuvant treatment.

Lipid composition can be measured using biochemical extraction methods for gas chromatography [15], but suffering from invasiveness, labour-intensive procedures and high cost [16]. Magnetic resonance spectroscopy (MRS) is a powerful tool for non-invasive biochemical quantification with scanners readily available in tertiary hospitals, but unable to discern lipid constituents due to overlapping signals on a onedimensional spectrum [17]. Localised correlation spectroscopy (L-COSY) is capable of discerning lipid constituents in breast cancer on a two-dimensional spectral map [18], but suffering from water contamination signal and limited accuracy resulted from dispersed peak pattern. Double quantum filtering (DQF), utilising signal selection targeting specific double bonds in molecular structure [19], effectively suppresses contamination signal and creates focused spectral peak. Hence, DQF-MRS can single out PUFA for high accuracy quantification [17], necessary in tumour due to low PUFA fraction, while DQF-COSY provides accurate quantification of lipid composition [19].

We therefore hypothesise there is a difference in lipid composition derived from DQF-MRS and DQF-COSY between LVI status, and is independent from cell division and inflammation in breast cancer.

\section{Materials and methods}

We conducted a two-group cross-sectional study to quantify lipid composition in whole tumours freshly excised from patients with breast cancer, with further comparison against histopathological findings (Fig. 1).

Study approval The study was approved by the North West Greater Manchester East Research Ethics Committee (REC Reference: 16/NW/0032), and signed written informed consent was obtained from the patients prior to entry into the study.

Clinical procedure Thirty female patients (age 39-78) with grade II or III invasive ductal carcinoma participated in the study. Patients over the age of 18 , undergoing a wide local excision or mastectomy, and with a tumour size larger than $1 \mathrm{~cm}$ in diameter on mammography were eligible. Patients with previous breast malignancies or patients who had neoadjuvant chemotherapy or neoadjuvant hormonal therapy prior to surgery were excluded. In total, 50 patients consented to be included in the study from 53 consecutive patients in the Breast Unit at Aberdeen Royal Infirmary. However, from these 50 resected tumour specimens, 11 were not scanned due to theatre delays and MRI scanner unavailability. A further 9 tumour specimens were excluded due to lobular phenotype $(n=2)$, mixed phenotype $(n=5)$ or grade $\mathrm{I}(n=2)$ on final histopathological examination of the excised tumour. Standard histopathological examination was performed to determine tumour size, grade, LVI, lymph node metastasis, receptors and Nottingham Prognostic Index (NPI) [20]. Upon full histological examination, there were 13 LVI negative and 17 LVI positive breast cancers. Immunostaining was conducted in a single batch for Ki-67 (Clone MIB-1, Dako, Dilution 1/100) and serotonin (Clone HTR1A, Thermo Fisher Scientific, Dilution 1/500) including appropriate positive controls, with immunostainer Ventana Benchmark XT with Ultraview DAB Detection Kit (760-500) (Ventana Medical Systems, Inc.), and assessed by a consultant pathologist. Ki67 was quantitatively assessed as percentage of positive nuclei $(0-100 \%)$ [21], while serotonin was semi-quantitatively assessed using the $\mathrm{H}$-score: Membranous staining was scored as 0 for 'no staining', $1+$ for 'weak staining', $2+$ for 'moderate staining' and $3+$ for 'strong staining'. The percentage of cells at different staining intensities was determined by visual assessment. The final score was calculated using the formula 1 $\times(\%$ of $1+$ cells $)+2 \times(\%$ of $2+$ cells $)+3 \times(\%$ of $3+$ cells $)$ [22].

Lipid composition The freshly excised tumour specimen without formalin treatment was immediately transported to the Aberdeen Biomedical Imaging Centre. The lipid composition spectra were acquired from a single voxel snug fit to the 
Fig. 1 Study design. A two-group cross-sectional study is shown in a flow chart. Fifty-three female breast cancer patients were found to be eligible at initial screening. Upon approach, three patients declined participation and the rest were consented to the study. After wide local excision or mastectomy, the freshly excised tumour specimen was scanned on a $3 \mathrm{~T}$ clinical MRI scanner to derive lipid composition using double quantum-filtered (DQF)MRS and DQF-correlation spectroscopy (DQF-COSY). Immunohistochemical examinations were conducted to assess lymphovascular invasion (LVI), Ki-67 and serotonin expression and Nottingham Prognostic Index (NPI). In total, 30 patients with invasive ductal carcinoma (IDC), 13 with LVI negative and 17 with LVI positive, participated in the study



tumour on a $3 \mathrm{~T}$ whole-body clinical MRI scanner (Achieva TX, Philips Healthcare) using a 32-channel receiver coil for high sensitivity detection and a body coil for uniform transmission. PUFA spectrum was acquired using DQF-MRS sequence [17] with a repetition time (TR) of $1250 \mathrm{~ms}$, echo time (TE) of $130 \mathrm{~ms}$, spectral editing frequency at $2.8 \mathrm{ppm}$, bandwidth of $2000 \mathrm{~Hz}$ and 1024 points. Reference spectrum (conventional MRS) without water suppression was acquired using PRESS sequence [23] with TR/TE of 1250/130 ms. Lipid spectral map was acquired using DQF-COSY sequence [19], with TR of $552 \mathrm{~ms}$, initial TE of $25 \mathrm{~ms}$, a $t_{1}$ increment of $1 \mathrm{~ms}, 256$ increments, bandwidth of $1000 \mathrm{~Hz}$ and 256 points. The acquisition durations were $10 \mathrm{~min} 40 \mathrm{~s}$ for DQF-MRS and 9 min $45 \mathrm{~s}$ for DQF-COSY. Further details of the methodology can be found in the Supplementary Material.

DQF-MRS and DQF-COSY are complementary methods for lipid composition quantification, with respective strength. DQF-MRS targets a single metabolite through the manipulation of quantum states of coupled spins, with the resonance frequency at $2.8 \mathrm{ppm}$ and $5.3 \mathrm{ppm}$ for PUFA [17]. The employment of the field gradient effectively eliminates background signals beyond target metabolite, while the binary nature of quantum coherence pathway selection leads to a $50 \%$ loss of the signal [17]. DQF-COSY and L-COSY are localised derivatives from COSY for $2 \mathrm{D}$ spectroscopy to allow the identification of molecular structures. L-COSY, without DQF, suffers from overwhelming background signal and broad spectral peak appearance [19]. DQF-COSY embeds DQF component within COSY, allowing suppression of uncoupled signal across the spectral domain and a sharp spectral peak appearance. However, DQF-COSY only retains $25 \%$ of the signal, as a consequence of the quantum coherence pathway selection and the use of stimulated echo [19].

PUFA was quantified using DQF-MRS and conventional MRS, demanding minimal discrepancies between the two acquisitions for accuracy. Both acquisitions adopted PRESS sequence backbone $[17,23]$ with identical voxel localisation RF pulse shape and field gradient strength for identical voxel location, voxel profile and chemical shift displacement. Both acquisitions also adopted identical receiver gain configuration with a shared scanner setup procedure for comparable nominal values and minimal hardware variability.

Lipid constituents were calculated as a ratio of the corresponding spectral peak against the methyl proton peak, without the quantification of absolute concentration using estimated $\mathrm{T}_{1}$ and $\mathrm{T}_{2}$. PUFA, quantified as spectral peak amplitude at $5.3 \mathrm{pm}$ from DQF-MRS, was referenced to $0.9 \mathrm{ppm}$ from conventional MRS, while all the other lipid constituents, quantified as corresponding spectral peak volumes from DQF-COSY, were referenced to $(0.9,0.9)$ ppm from DQFCOSY. The PUFA peak at 5.3 ppm from DQF-MRS is spectrally edited through coupling peak at $2.8 \mathrm{ppm}$ [17] equivalent to the cross peak at $(5.3,2.8) \mathrm{ppm}$ in DQF-COSY or $2.8 \mathrm{ppm}$ in conventional $1 \mathrm{D} \mathrm{MRS}$, with the ratio against methyl proton 
proportional to PUFA fraction [24]. The unsaturated fatty acids (UFA) at $(2.1,2.1) \mathrm{ppm}$ from DQF-COSY encompasses PUFA and MUFA substrates manifested at $(5.3,2.8) \mathrm{ppm}$ and $(5.3,2.1) \mathrm{ppm}$ respectively, with the ratio of UFA against methyl proton negatively proportional to SFA fraction (SFA $=1$-UFA/methyl) $[25,26]$.

The peak amplitudes of PUFA (5.3 ppm) and methyl proton $(0.9 \mathrm{ppm})$ were quantified using prior knowledge databases [27] and AMARES algorithm [28] in the jMRUI software (v3.0, TRANSACT) [29]. The peak volumes of UFA, triglycerides (TRG) and MUFA were quantified at $(2.1,2.1)$ ppm, $(4.3,4.3) \mathrm{ppm}$ and $(5.3,2.1) \mathrm{ppm}$ respectively. Lipid spectral maps were quantified in the Felix software (v2007, Accelrys Inc.), with zerofilling to 512 points and twodimensional sine bell apodisation [19].

Statistical analysis All statistical analysis was performed in the SPSS software (Release 23.0, SPSS Inc.). Normality was determined on all the collected data using the Shapiro-Wilk test. Mann-Whitney $U$ tests were used for MUFA, SFA and TRG while independent sample $t$ test for PUFA (normally distributed) between LVI groups. Fisher's exact tests were applied for categorical variables (clinicopathological features). Spearman's correlation tests were performed between lipid composition against proliferative activity marker Ki-67 and serotonin, while Pearson's correlation test between PUFA and serotonin in the entire cohort. A $p$ value $<0.05$ was accepted as being statistically significant.

\section{Results}

The patient demographics are summarised in Table 1. There were no significant differences in age and body mass index (BMI) between groups. There were no significant differences in tumour size, NPI or stage between groups.

There were significantly lower MUFA $(z=2.3470, p=$ 0.0189 , Fig. 2a, Table 2) in LVI positive (median: 0.37, interquartile range (IQR): $0.25-0.64)$ than LVI negative $(0.63$, $0.49-0.96)$. There were significantly lower TRG $(z=$ $2.2810, p=0.0226$, Fig. 2b, Table 2$)$ in LVI positive (1.32, $0.95-2.43)$ than LVI negative $(2.5,1.92-4.15)$. There was no significant difference in SFA ( $p=0.6009$, Fig. $2 c$, Table 2$)$ between LVI negative $(0.50,0.36-0.57)$ and LVI positive $(0.53,0.41-0.58)$. There was no significant difference in PUFA ( $p=0.1641$, Fig. 2 d, Table 2 ) between LVI negative $(0.013 \pm 0.006)$ and LVI positive $(0.010 \pm 0.006)$.

There were no significant correlations between lipid composition against Ki-67 (Fig. 3, Table 2). There were no significant correlations between MUFA, TRG and SFA against serotonin, but a borderline significant negative correlation between PUFA and serotonin $(r=-0.3616, p=0.0496$, Fig. 4, Table 2).

\section{Discussion}

In this work, we found positive LVI in breast cancer associated with lower MUFA and triglycerides, but not with SFA or PUFA. Lipid compositions were not correlated with serotonin or proliferative activity marker Ki-67, apart from a borderline negative correlation between PUFA and serotonin. Hence, lipid composition in breast tumour might be a potential marker of LVI.

LVI positivity was negatively associated with MUFA and TRG, but not SFA or PUFA. SFA, the main product of de novo lipogenesis, showed no significant difference between LVI status, indicating potential negative feedback to avoid apoptosis [30] and impaired angiogenesis [31] arising from a further increase in SFA. SFA, an essential building block for cell structural growth, is tightly regulated in the endogenous fatty acid metabolism [32]. Excess SFA palmitate inhibits the mitochondrial phospholipid cardiolipin [30] and suppresses the activity of cathepsin critical for the formation of new endothelial cell-lined blood vessels and consequent cancer proliferation [31]. Hence, the equal spread of SFA distribution in LVI positive and LVI negative might indicate a common protective mechanism against lipotoxicity [30]. The increased MUFA in negative LVI indicates an accelerated conversion from excess SFA for membrane phospholipid synthesis [32] and epidermal growth factor signalling [33], while a reduced MUFA observed in positive LVI due to the export of SFA via tumour cells crossing the lymphatic vasculature [11].

The reduced TRG in positive LVI indicates accelerated de novo lipogenesis unconstrained from lipotoxicity mitigated through SFA clearance via tumour cells crossing the lymphatic vasculature. Triglycerides (TRG), occupying spectral peak at $(4.3,4.3) \mathrm{ppm}$ [18], typically maintain a constant ratio against methyl proton since the ratio of head groups and methyl groups in the TRG chains is fixed. However, TRG is broken down into glycerides and fatty acids in tumours under lipolytic enzyme overexpression [34], with the variation in observation primarily attributed to the glycerides. The spectral peak at $(4.3,4.3) \mathrm{ppm}$ has low SNR in low-fat fraction tumours [35, $36]$ and the contamination signal from mono- and diglycerides at $3 \mathrm{~T}[24,37]$ may affect experimental accuracy. PUFA showed no association with LVI, indicating the depletion of naturally low abundant PUFA for membrane synthesis [17] remaining unresolved despite the potential import from peri-tumoural adipocytes in positive LVI. Hence, lipid composition is associated with LVI through the amplification of de novo lipogenesis and could impact on peri-tumoural adipocyte lipid content [38].

Lipid composition did not show a significant correlation against proliferative activity marker Ki-67, indicating no evidence to support a direct link between lipids and tumour cell division within the framework of this work. Lipid composition did not show a significant correlation, apart from a borderline 
Table 1 Clinical characteristics of patients. Descriptive statistics of breast cancer patients with histopathological findings are shown for each group and the entire cohort. Numbers are expressed as mean and standard deviation (apart from Nottingham Prognostic Index where median and interquartile range are shown), with pathological entries expressed as number of positive observations

\begin{tabular}{|c|c|c|c|c|}
\hline \multirow[t]{2}{*}{ Characteristic } & \multirow[t]{2}{*}{ All $(n=30)$} & \multicolumn{2}{|c|}{ Lymphovascular invasion (LVI) } & \multirow[t]{2}{*}{$p$ value } \\
\hline & & Negative $(n=13)$ & Positive $(n=17)$ & \\
\hline Age (years) & $61.1 \pm 11.5$ & $59.2 \pm 12.6$ & $62.6 \pm 10.6$ & 0.4375 \\
\hline Body mass index (BMI) & $30.4 \pm 6.4$ & $31.2 \pm 7.5$ & $29.5 \pm 5.4$ & 0.5451 \\
\hline Tumour size $(\mathrm{cm})$ & $2.5 \pm 0.8$ & $2.4 \pm 0.7$ & $2.5 \pm 0.8$ & 0.6070 \\
\hline Nottingham Prognostic Index (NPI) & $4.41(3.62-4.56)$ & $4.29(3.62-4.50)$ & $4.44(3.70-4.60)$ & 0.4764 \\
\hline \multicolumn{5}{|l|}{ Tumour features } \\
\hline \multicolumn{5}{|l|}{ pTNM Stage } \\
\hline $\mathrm{I}$ & 5 & 3 & 2 & \multirow[t]{2}{*}{0.628} \\
\hline II & 25 & 10 & 15 & \\
\hline \multicolumn{5}{|l|}{ Histological grade } \\
\hline II & 15 & 6 & 9 & \multirow[t]{2}{*}{1.000} \\
\hline III & 15 & 7 & 8 & \\
\hline Lymph node involvement & 8 & 2 & 6 & 0.407 \\
\hline Oestrogen receptor $(\mathrm{ER}+)$ & 22 & 8 & 14 & 0.242 \\
\hline Human epidermal growth factor receptor 2 (HER2+) & 6 & 2 & 4 & 0.672 \\
\hline Triple-negative breast cancer (TNBC) & 7 & 5 & 2 & 0.190 \\
\hline
\end{tabular}

negative correlation in PUFA, against serotonin (5-HT), an epigenetically identified marker of poor 10-year survival rate [14]. Hence, serotonin primarily reflects the upregulation in membrane synthesis [13] (more depleted PUFA [17]) induced by environmental factors, rather than intrinsic aggressiveness or rapid division of the tumour cells.

Lipid composition in the breast has been quantified using MRS in the adipose tissue from both patients and healthy volunteers. Conventional STEAM sequence has been used for the quantification of lipid composition in breast adipose tissue from patients at $3 \mathrm{~T}$ [39] and healthy volunteers at $7 \mathrm{~T}$ [40]. L-COSY has been used to reveal elevated PUFA in breast adipose tissue in BRCA1 gene mutations carriers compared to healthy controls [18]. Spatially resolved MRS using multiple gradient-echo imaging, an extension from the Dixon method, revealed elevated peri-tumoural SFA and suppressed
Table 2 Lipid composition and histological markers Ki-67 and serotonin in lymphovascular invasion (LVI) negative and positive breast tumours. Correlation scores (Spearman's rho and Pearson's $r$ ) of lipid composition, quantified in double quantum-filtered correlation spectroscopy (DQF-COSY) and DQF magnetic resonance spectroscopy (DQF-MRS), against Ki-67 and serotonin are also shown.
Monounsaturated, saturated fatty acids (MUFA, SFA) and triglycerides (TRG) were quantified from DQF-COSY, while polyunsaturated FA (PUFA) from DQF-MRS. The spread of lipid composition from DQFCOSY and Ki-67 expression is non-normal and is reported as median and interquartile range (IQR). Significant findings $(p<0.05)$ are marked by '*,

\begin{tabular}{|c|c|c|c|c|c|c|}
\hline \multirow[t]{2}{*}{ LVI group } & \multicolumn{3}{|l|}{ DQF-COSY } & \multirow{2}{*}{$\begin{array}{l}\text { DQF-MRS } \\
\text { PUFA }\end{array}$} & \multicolumn{2}{|c|}{ Histological markers } \\
\hline & SFA & MUFA & TRG & & $\mathrm{Ki}-67(\%)$ & Serotonin $(0-300)$ \\
\hline All $(n=30)$ & $0.52(0.36-0.58)$ & $0.49(0.37-0.69)$ & $2.01(1.18-2.99)$ & $0.011 \pm 0.007$ & $16.6(9.7-24.0)$ & $107 \pm 62$ \\
\hline LVI negative ( $n=13$ ) & $0.50(0.36-0.57)$ & $0.63(0.49-0.96)^{\mathrm{a}}$ & $2.5(1.92-4.15)$ & $0.013 \pm 0.006$ & $17.3(11.6-29.1)$ & $84 \pm 48$ \\
\hline LVI positive ( $n=17)$ & $0.53(0.41-0.58)$ & $0.37(0.25-0.64)$ & $1.32(0.95-2.43)$ & $0.010 \pm 0.006$ & $15.3(9.6-22.9)$ & $124 \pm 68$ \\
\hline $\begin{array}{l}t / z \text {-score } \\
p \text { value }\end{array}$ & $\begin{array}{l}z=0.5230 \\
p=0.6009\end{array}$ & $\begin{array}{l}z=2.3470 \\
p=0.0189^{*}\end{array}$ & $\begin{array}{l}z=2.2810 \\
p=0.0226^{*}\end{array}$ & $\begin{array}{l}t=1.4319 \\
p=0.1641\end{array}$ & $\begin{array}{l}\mathrm{z}=0.7320 \\
p=0.4639\end{array}$ & $\begin{array}{l}t=1.9255 \\
p=0.0644\end{array}$ \\
\hline \multicolumn{7}{|c|}{ Correlation $(r / \rho$-score, $p$ value $)$} \\
\hline $\mathrm{Ki}-67$ & $0.2254,0.2312$ & $0.3108,0.1008$ & $0.0621,0.7445$ & $0.2071,0.2721$ & & \\
\hline Serotonin & $-0.1444,0.4465$ & $0.0212,0.9129$ & $0.1312,0.4894$ & $-0.3616,0.0496^{*}$ & & \\
\hline
\end{tabular}

${ }^{a}$ MUFA from one LVI negative breast tumour was not quantifiable due to low signal-to-noise ratio, i.e., $n=12$ 
Fig. 2 Dot plots to show the group difference in monounsaturated fatty acids (MUFA) $(n=12,17)(\mathbf{a})$, triglycerides (TRG) $(n=13,17)$ (b), saturated FA (SFA) $(n=13$, 17) (c) and polyunsaturated FA (PUFA) $(n=13,17)$ (d) between LVI negative and LVI positive breast tumours. The error bar indicates the median and interquartile range (mean $\pm \mathrm{SD}$ tests $(\mathbf{a}-\mathbf{c})$ and independent sample $t$ test (d) were performed between the groups and $p$ value is shown for each plot. Statistically significant $p$ values $(<0.05)$ are marked by an asterisk for PUFA). Mann-Whitney $U$
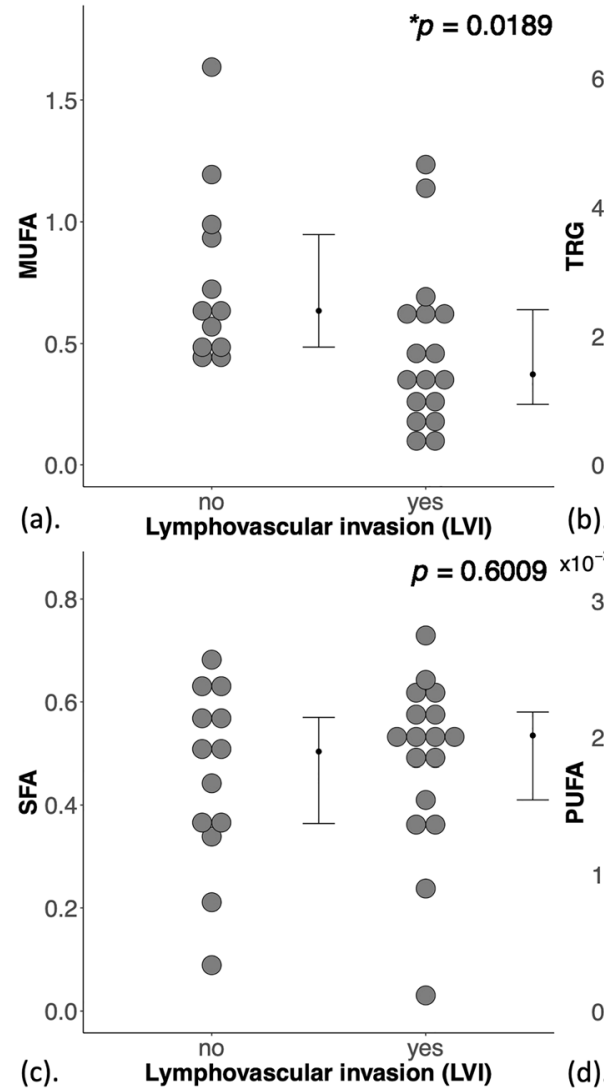

(c). Lymphovascular invasion (LVI)

$\begin{array}{cc}\text { no } & \text { yes } \\ \text { (d). } & \text { Lymphovascular invasion (LVI) }\end{array}$

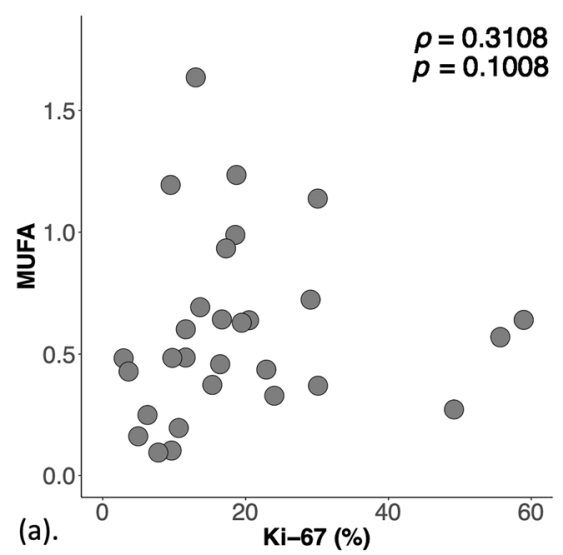

(b).

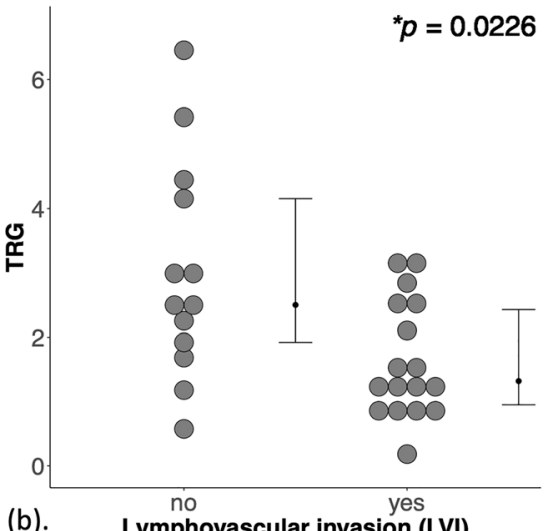

Lymphovascular invasion (LVI)

$p=0.1641$

0



Fig. 3 Scatter plots to show the correlation of monounsaturated fatty acids (MUFA) $(n=29)(\mathbf{a})$, triglycerides (TRG) $(n=30)(\mathbf{b})$, saturated FA (SFA) $(n=30)(\mathbf{c})$, and polyunsaturated FA (PUFA) $(n=30)(\mathbf{d})$ with $\mathrm{Ki}-67$ expression within the entire cohort.

Spearman's rank correlation tests were performed and rho $(\rho)$ scores and $p$ values are displayed. There were no significant correlations between MUFA, TRG, SFA or PUFA with Ki-67 expression

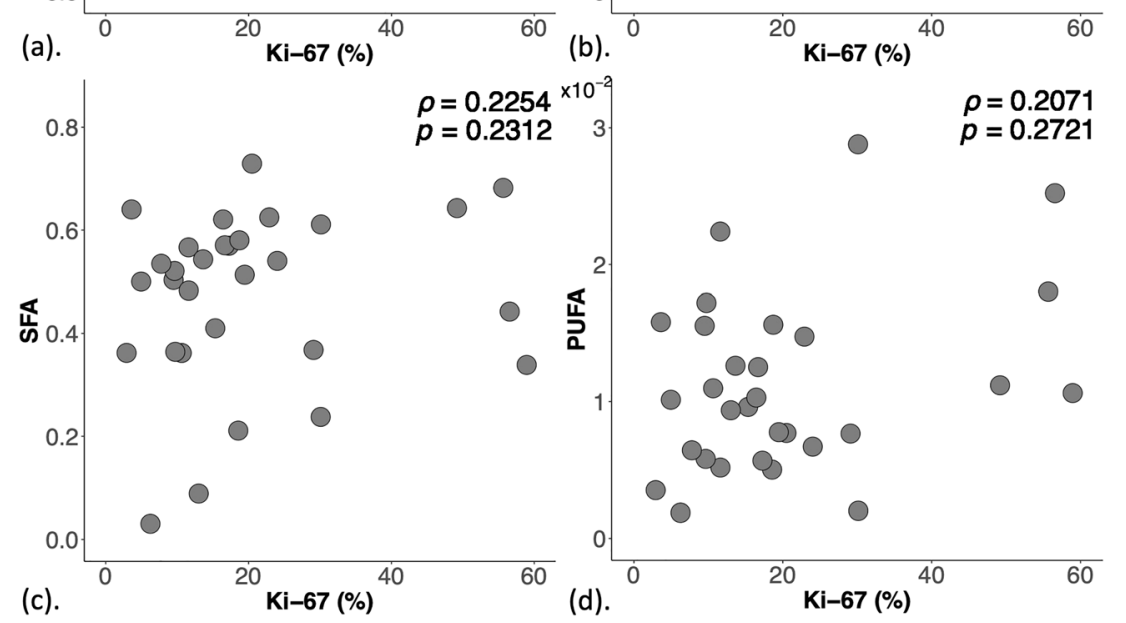


Fig. 4 Scatter plots to show the correlation of monounsaturated fatty acids (MUFA) $(n=29)$ (a), triglycerides (TRG) $(n=30)(\mathbf{b})$, saturated FA (SFA) $(n=30)(\mathbf{c})$ and polyunsaturated FA (PUFA) $(n=30)$ (d) with serotonin expression within the entire cohort. Spearman's rank correlation $(\mathbf{a}-\mathbf{c})$ and Pearson's correlation (d) tests were performed and corresponding rho $(\rho)$ and $r$ scores and $p$ values are displayed. Statistically significant $p$ values $(<0.05)$ are marked by an asterisk
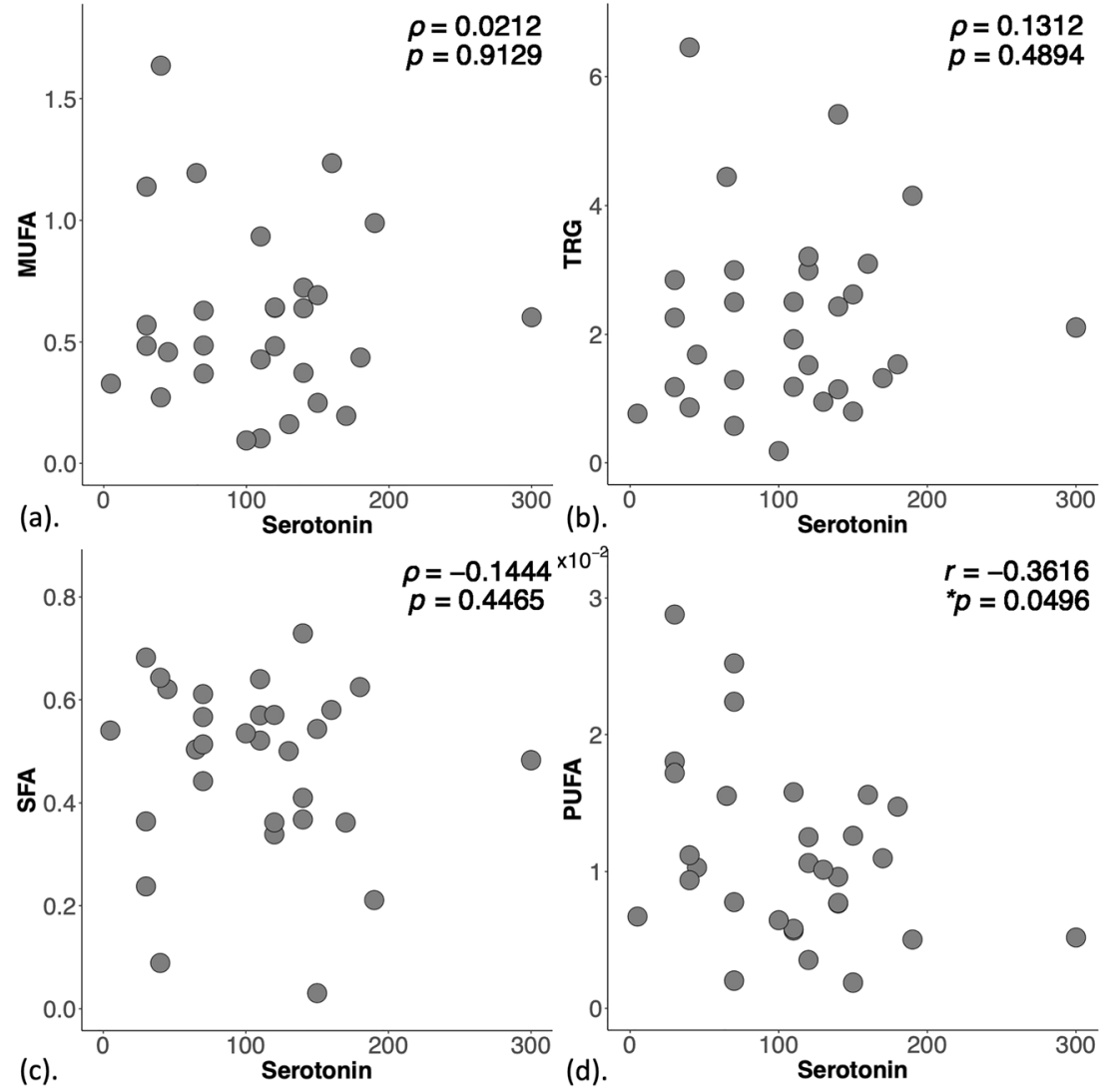

MUFA in postmenopausal patients compared to healthy controls [41, 42]. However, the investigation of intra-tumoural lipid composition has been limited to fat fraction owing to low abundance of lipids inside breast tumour [43], with the feasibility as a potential marker for response to chemotherapy [35] and the identification of tumour subtypes [36] attempted. The introduction of DQF-MRS allowed the observation of substantially reduced PUFA in breast tumours [17], and this work augmented the understanding of lipid composition in breast cancer [44] using the most sensitive MRS approaches through a formal clinical study on LVI.

This study is the first to investigate the association between lipid composition and LVI positivity in whole human breast tumours. The quantitative nature of the novel spectroscopy method provides an objective approach for LVI evaluation, compared to time-consuming and experience-dependent histological approaches. The quantification at whole tumour estimates an overall degree of compromise in lymphatic vasculature [11], eliminating partial sampling error inherent in biopsy approaches (high false negative of $40 \%$ in this study alone) [7]. The ex vivo study design allows the elimination of biological noise and employment for accurate quantification, particularly in the context of depleted PUFA and triglycerides. The deployment of DQF approach suppresses contamination signals proximal to PUFA and triglycerides in the spectral domain, further enhancing quantification accuracy.
The feasibility of the employed MRS approaches for in vivo application has been demonstrated using standard clinical scanner, while the clinical utility with underpinning sensitivity to critical tumour metabolic pathways has been shown as an initial step in clinical translation. The lipid composition coupled with current clinical dynamic contrast-enhanced (DCE) and diffusion-weighted MRI may enhance the diagnostic accuracy to reduce false-positive rate.

SFA and TRG are determined from diagonal peaks, with MUFA from cross peak, leading to a difference in scaling from underlying population in energy states. Hence, the comparison is performed on individual lipid constituent across patients, and comparison of nominal values between peaks will require further correction on the scaling factors. The overlap of the distribution in MUFA or TRG between groups may limit the diagnostic value of lipid composition as a biomarker in LVI. This work, as a precursor for consequent in vivo patient studies, attempted to augment the understanding of lipid composition in breast cancer with limited statistical power for multiple comparison correction. Future large cohort in vivo studies, supported by novel spectral analysis approaches [45], are required to confirm the relationship between lipid composition and LVI. Further trials integrating the lipid composition for treatment planning are critical, since neoadjuvant chemotherapy is more likely to be recommended for LVI positive patients [46]. 
In conclusion, MUFA and triglycerides are reduced in positive LVI resulting from accelerated de novo lipogenesis. Lipid composition observed through novel spectroscopy method might provide a biomarker to study LVI in breast cancer.

Supplementary Information The online version contains supplementary material available at https://doi.org/10.1007/s00330-020-07502-4.

Acknowledgements The authors would like to thank Dr. Nicholas Senn for conducting data auditing, Dr. Matthew Clemence (Philips Healthcare Clinical Science, UK) for clinical scientist support, Dr. Tim Smith for biologist support, Mr. Gordon Buchan for technician support, Ms Bolanle Brikinns for patient recruitment support, Ms Dawn Younie for logistic support and Prof. Andrew M. Blamire for advice on MRS. The authors would also like to thank Mr Roger Bourne and Ms Mairi Fuller for providing access to the patients.

Funding This study has received funding from Friends of Aberdeen and North Centre for Haematology, Oncology and Radiotherapy (ANCHOR) (RS2015 004). Sai Man Cheung's PhD study was jointly supported by Elphinstone scholarship, Roland Sutton Academic Trust and John Mallard scholarship.

\section{Compliance with ethical standards}

Guarantor The scientific guarantor of this publication is Dr. Jiabao He.

Conflict of interest The authors of this manuscript declare no relationships with any companies, whose products or services may be related to the subject matter of the article.

Statistics and biometry No complex statistical methods were necessary for this paper.

Informed consent Only if the study is on human subjects:

Written informed consent was obtained from all subjects (patients) in this study.

Ethical approval National Health Service Research Ethics Committee approval was obtained.

\section{Methodology \\ - prospective \\ - cross-sectional study \\ - performed at one institution}

Open Access This article is licensed under a Creative Commons Attribution 4.0 International License, which permits use, sharing, adaptation, distribution and reproduction in any medium or format, as long as you give appropriate credit to the original author(s) and the source, provide a link to the Creative Commons licence, and indicate if changes were made. The images or other third party material in this article are included in the article's Creative Commons licence, unless indicated otherwise in a credit line to the material. If material is not included in the article's Creative Commons licence and your intended use is not permitted by statutory regulation or exceeds the permitted use, you will need to obtain permission directly from the copyright holder. To view a copy of this licence, visit http://creativecommons.org/licenses/by/4.0/.

\section{References}

1. Cancer Research UK (2020) Breast cancer statistics. Available from: https://www.cancerresearchuk.org/health-professional/ cancer-statistics/statistics-by-cancer-type/breast-cancer/survival\# ref-. Accessed: 21st July, 2020

2. Cuzick J, Sestak I, Baum M et al (2010) Effect of anastrozole and tamoxifen as adjuvant treatment for early-stage breast cancer: 10 year analysis of the ATAC trial. Lancet Oncol 11:1135-1141

3. Rakha EA, Martin S, Lee AHS et al (2012) The prognostic significance of lymphovascular invasion in invasive breast carcinoma. Cancer 118:3670-3680

4. Pan H, Gray R, Braybrooke J et al (2017) 20-year risks of breastcancer recurrence after stopping endocrine therapy at 5 years. $\mathrm{N}$ Engl J Med 377:1836-1846

5. Sahoo S, Lester SC (2009) Pathology of breast carcinomas after neoadjuvant chemotherapy: an overview with recommendations on specimen processing and reporting. Arch Pathol Lab Med 133: 633-642

6. Sharifi S, Peterson MK, Baum JK, Raza S, Schnitt SJ (1999) Assessment of pathologic prognostic factors in breast core needle biopsies. Mod Pathol 12:941-945

7. Usami S, Moriya T, Kasajima A et al (2005) Pathological aspects of core needle biopsy for non-palpable breast lesions. Breast Cancer $12: 272-278$

8. Zaorsky NG, Patil N, Freedman GM, Tuluc M (2012) Differentiating lymphovascular invasion from retraction artifact on histological specimen of breast carcinoma and their implications on prognosis. J Breast Cancer 15:478-483

9. Koo JS, Jung W-H, Kim H (2010) Epithelial displacement into the lymphovascular space can be seen in breast core needle biopsy specimens. Am J Clin Pathol 133:781-787

10. Ozmen V, Karanlik H, Cabioglu N et al (2006) Factors predicting the sentinel and non-sentinel lymph node metastases in breast cancer. Breast Cancer Res Treat 95:1-6

11. Christiansen A, Detmar M (2011) Lymphangiogenesis and cancer. Genes Cancer 2:1146-1158

12. Klahan S, Wong HS-C, Tu S-H et al (2017) Identification of genes and pathways related to lymphovascular invasion in breast cancer patients: a bioinformatics analysis of gene expression profiles. Tumor Biol 39:101042831770557

13. Herr N, Bode C, Duerschmied D (2017) The effects of serotonin in immune cells. Front Cardiovasc Med 4:48

14. Leoncikas V, Wu H, Ward LT, Kierzek AM, Plant NJ (2016) Generation of 2,000 breast cancer metabolic landscapes reveals a poor prognosis group with active serotonin production. Sci Rep 6: 19771

15. Bagga D, Anders KH, Wang H-J, Glaspy JA (2002) Long-chain n3-to-n-6 polyunsaturated fatty acid ratios in breast adipose tissue from women with and without breast cancer. Nutr Cancer 42:180 185

16. Kang JX, Wang J (2005) A simplified method for analysis of polyunsaturated fatty acids. BMC Biochem 6:5

17. He Q, Shkarin P, Hooley RJ, Lannin DR, Weinreb JC, Bossuyt VIJ (2007) In vivo MR spectroscopic imaging of polyunsaturated fatty acids (PUFA) in healthy and cancerous breast tissues by selective multiple-quantum coherence transfer (Sel-MQC): a preliminary study. Magn Reson Med 58:1079-1085

18. Ramadan S, Arm J, Silcock J et al (2005) Lipid and metabolite deregulation in the breast tissue of women carrying BRCA1 and BRCA2 genetic mutations. Radiology 275:675-682

19. Prescot AP, Dzik-Jurasz ASK, Leach MO, Sirohi B, Powles R, Collins DJ (2005) Localized COSY and DQF-COSY ${ }^{1} \mathrm{H}-\mathrm{MRS}$ sequences for investigating human tibial bone marrow in vivo and 
initial application to patients with acute leukemia. J Magn Reson Imaging 22:541-548

20. Elston CW, Ellis IO (1991) Pathological prognostic factors in breast cancer. I. The value of histological grade in breast cancer: experience from a large study with long-term follow-up. Histopathol 19: 403-410

21. Tuominen VJ, Ruotoistenmäki S, Viitanen A, Jumppanen M, Isola J (2010) ImmunoRatio: a publicly available web application for quantitative image analysis of estrogen receptor (ER), progesterone receptor (PR), and Ki-67. Breast Cancer Res 12:R56

22. McCarty KS Jr, Miller LS, Cox EB, Konrath J, McCarty KS Sr (1985) Estrogen receptor analyses. Correlation of biochemical and immunohistochemical methods using monoclonal antireceptor antibodies. Arch Pathol Lab Med 109:716-721

23. Bottomley PA (1987) Spatial localization in NMR spectroscopy. Ann N Y Acad Sci 508:333-348

24. Zancanaro C, Nano R, Marchioro C, Sbarbati A, Boicelli A, Osculati F (1994) Magnetic resonance spectroscopy investigations of brown adipose tissue and isolated brown adipocytes. J Lipid Res 35:2191-2199

25. Knothe G, Kenar JA (2004) Determination of the fatty acid profile by ${ }^{1} \mathrm{H}-\mathrm{NMR}$ spectroscopy. Eur J Lipid Sci Technol 106:88-96

26. Corbin IR, Furth EE, Pickup S, Siegelman ES, Delikatny EJ (2009) In vivo assessment of hepatic triglycerides in murine non-alcoholic fatty liver disease using magnetic resonance spectroscopy. Biochim Biophys Acta 1791:757-763

27. Lundbom J, hakkarainen A, Fielding B et al (2010) Characterizing human adipose tissue lipids by long echo time ${ }^{1} \mathrm{H}-\mathrm{MRS}$ in vivo at 1.5 Tesla: validation by gas chromatography. NMR Biomed 23: 466-472

28. Vanhamme L, van den Boogaart A (1997) Improved method for accurate and efficient quantification of MRS data with use of prior knowledge. J Magn Reson 129:35-43

29. Naressi A, Couturier C, Castang I, de Beer R, Graveron-Demilly D (2001) Java-based graphical user interface for MRUI, a software package for quantitation of in vivo/medical magnetic resonance spectroscopy signals. Comput Biol Med 31:269-286

30. Hardy S, El-Assaad W, Przybytkowski E, Joly E, Prentki M, Langelier Y (2003) Saturated fatty acid-induced apoptosis in MDA-MB-231 breast cancer cells. J Biol Chem 278:31861-31870

31. Zhang J, Shan Y, Li Y, Luo X, Shi H (2017) Palmitate impairs angiogenesis via suppression of cathepsin activity. Mol Med Rep 15:3644-3650

32. Menendez JA, Lupu R (2007) Fatty acid synthase and the lipogenic phenotype in cancer pathogenesis. Nat Rev Cancer 7:763-777

33. Nagahashi M, Ramachandran S, Kim EY et al (2012) Sphingosine1-phosphate produced by sphingosine kinase 1 promotes breast cancer progression by stimulating angiogenesis and lymphangiogenesis. Cancer Res 72:726-735
34. Nomura DK, Long JZ, Niessen S, Hoover HS, Ng S-W, Cravatt BF (2010) Monoacylglycerol lipase regulates a fatty acid network that promotes cancer pathogenesis. Cell 140:49-61

35. Jagannathan NR, Singh M, Govindaraju V et al (1998) Volume localized in vivo proton MR spectroscopy of breast carcinoma: variation of water-fat ratio in patients receiving chemotherapy. NMR Biomed 11:414-422

36. Agarwal K, Sharma U, Mathur S, Seenu V, Parshad R, Jagannathan NR (2018) Study of lipid metabolism by estimating the fat fraction in different breast tissues and in various breast tumor sub-types by in vivo ${ }^{1} \mathrm{H}$ MR spectroscopy. Magn Reson Imaging 49:116-122

37. Nieva-Echevarría B, Goicoechea E, Manzanos MJ, Guillén MD (2014) A method based on ${ }^{1} \mathrm{H}$ NMR spectral data useful to evaluate the hydrolysis level in complex lipid mixtures. Food Res Int 66: 379-387

38. Wang YY, Attané C, Milhas D et al (2017) Mammary adipocytes stimulate breast cancer invasion through metabolic remodeling of tumor cells. JCI Insight 2:1-20

39. Coum A, Ouldamer L, Noury F et al (2016) In vivo MR spectroscopy of human breast tissue: quantification of fatty acid composition at a clinical field strength (3 T). MAGMA 29:1-4

40. Dimitrov IE, Douglas D, Ren J et al (2012) In vivo determination of human breast fat composition by ${ }^{1} \mathrm{H}$ magnetic resonance spectroscopy at 7 T. Magn Reson Med 67:20-26

41. Freed M, Storey P, Lewin AA et al (2016) Evaluation of breast lipid composition in patients with benign tissue and cancer by using multiple gradient-echo MR imaging. Radiology 281:43-53

42. Lewin AA, Storey P, Moccaldi M, Moy L, Kim SG (2019) Fatty acid composition in mammary adipose tissue measured by gradientecho spectroscopic MRI and its association with breast cancer. Eur J Radiol 116:205-211

43. Fardanesh R, Marino MA, Avendano D, Leithner D, Pinker K, Thakur SB (2019) Proton MR spectroscopy in the breast: technical innovations and clinical applications. J Magn Reson Imaging 50: 1033-1046

44. Chhetri A, Li X, Rispoli JV (2020) Current and emerging magnetic resonance-based techniques for breast cancer. Front Med 7:175

45. Mallikourti V, Cheung SM, Gagliardi T, Masannat Y, Heys SD, He J (2019) Optimal phased-array signal combination for polyunsaturated fatty acids measurement in breast cancer using multiple quantum coherence MR spectroscopy at 3 T. Sci Rep 9:9259

46. Ragage F, Debled M, MacGrogan G et al (2010) Is it useful to detect lymphovascular invasion in lymph node-positive patients with primary operable breast cancer? Cancer 116:3093-3101

Publisher's note Springer Nature remains neutral with regard to jurisdictional claims in published maps and institutional affiliations. 